Document downloaded from:

http://hdl.handle.net/10251/40304

This paper must be cited as:

Areias, A.; Gómez-Tejedor, JA.; Sencadas, V.; Alio, J.; Gómez Ribelles, JL.; LancerosMendez, S. (2012). Assessment of Parameters Influencing fiber characteristics of chitosan nanofiber membrane to optimize fiber mat productioi. Polymer Engineering and Science. 52(6):1293-1300. doi:10.1002/pen.23070.

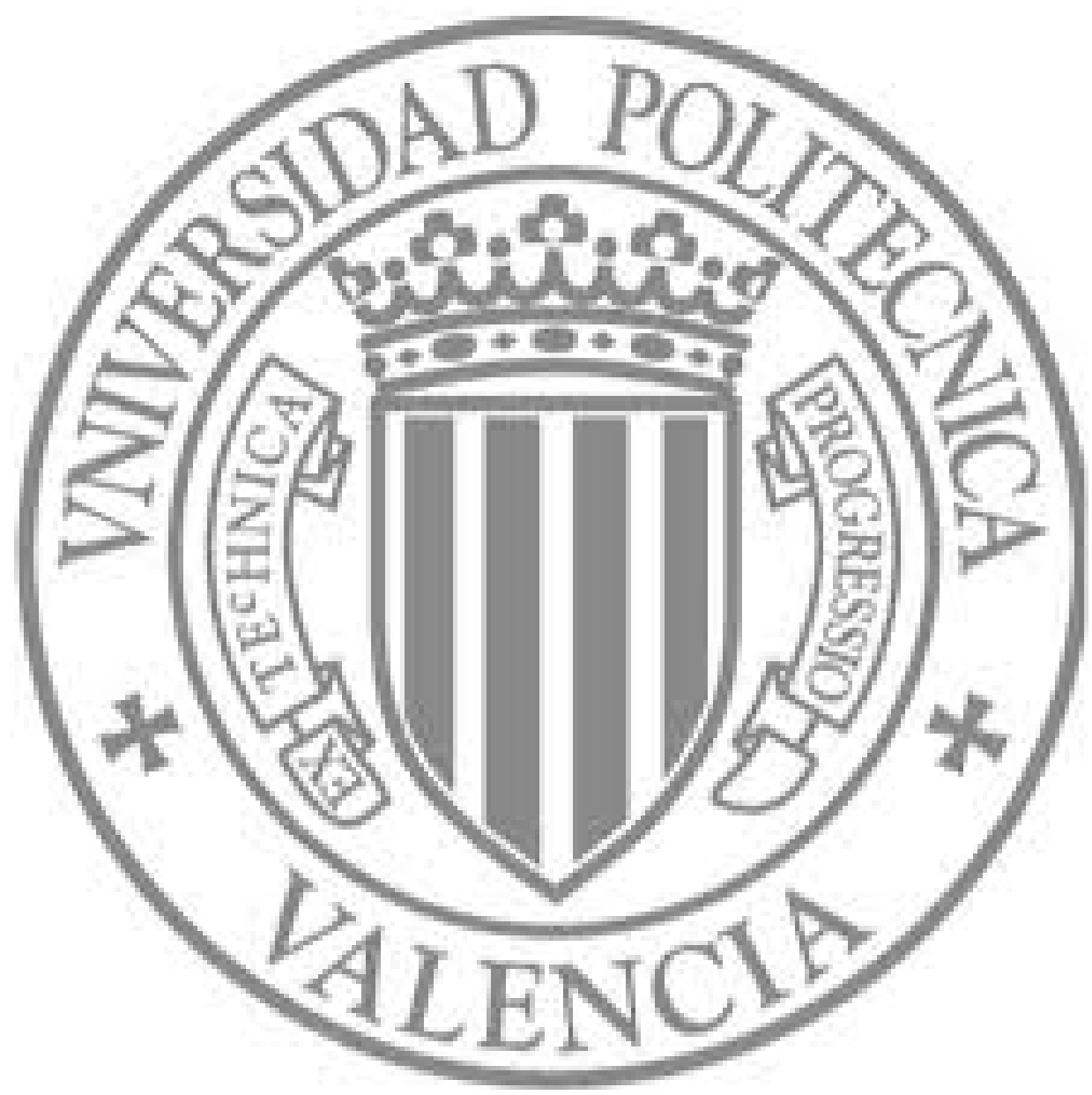

The final publication is available at

http://dx.doi.org/10.1002/pen.23070

Copyright Wiley 


\title{
Assessment of Parameters Influencing Fiber Characteristics of Chitosan Nanofiber Membrane in order to Optimize Fiber Mat Production
}

\author{
A. C. Areias ${ }^{1,2}$, J. A. Gómez-Tejedor ${ }^{2}$, V. Sencadas ${ }^{1}$, J. Alió ${ }^{3,4}$, J. L. Gómez Ribelles ${ }^{2,5,6}$ \\ and S. Lanceros-Mendez ${ }^{1}$ \\ ${ }^{1}$ Centro/Departamento de Física, Universidade do Minho, Campus de Gualtar, 4710- \\ 058 Braga, Portugal \\ ${ }^{2}$ Center for Biomaterials and Tissue Engineering, Universitat Politècnica de València, \\ Camino de Vera s/n, 46022 Valencia, Spain \\ ${ }^{3}$ Universidad Miguel Hernández de Alicante, Spain. \\ ${ }^{4}$ VISSUM Instituto Oftalmológico de Alicante, Spain. \\ ${ }^{5}$ Prince Felipe Research Center, Autopista del Saler 16, 46013 Valencia, Spain \\ ${ }^{6}$ Networking Research Center on Bioengineering, Biomaterials and Nanomedicine \\ (CIBER-BBN), Valencia, Spain
}

Email: jogomez@ fis.upv.es 


\begin{abstract}
Electrospun chitosan nanofiber mats have been obtained using chitosan solutions in a mixture of trifluoroacetic acid and dichloromethane. The relationship between processing parameters (solvent composition, polymer concentration in the solution, feeding rate, applied voltage, travelling distance between the needle and the collector) and fiber morphology was studied. Taguchi's methodology was followed to determine which parameters have the strongest influence on mean fiber diameter and fiber homogeneity. Chitosan nanofibers obtained with this procedure were water soluble due to the protonation of amine side groups but were successfully neutralized by immersion in calcium carbonate solutions. It was established that fiber diameter is mainly determined by the solution concentration and the distance from the needle to the collector while other parameters have less influence. The set of parameters that produce the thinnest fibers were a concentration of $8 \mathrm{wt} \%$, a TFA/DCM ratio of 80:20 (v/v \%), a voltage of $30 \mathrm{kV}$, a flow rate of $6.0 \mathrm{~mL} / \mathrm{h}$, a gap distance of $10 \mathrm{~cm}$, using a needle diameter of 0.5 , allowing to produce randomly oriented mats with a mean fiber thickness of $66 \mathrm{~nm}$.
\end{abstract}




\section{INTRODUCTION}

Biomaterials have been used in various scientific fields such as pharmacology [1], bioengineering [2] and electronics [3]. Tissue engineering techniques require a sufficient number of cells with the right phenotype to initiate tissue regeneration "in vitro" or "in vivo". The biomaterial that constitutes a culture substrate for expansion or differentiation is not merely a surface to which cells adhere. Cell adhesion mediated by proteins initiates a signaling cascade that is able to induce or modify essential pathways that determine cell response in such aspects as gene expression, proliferation or migration. Surface characteristics of the substrate highly determine cell response, and micro or nano-topography is one of the important parameters. It has been pointed out that a substrate consisting in materials analogous to those forming the natural extracellular matrix and in the form of nano- or sub-micron fibers might be favorable to induce particular responses in monolayer cultured cells. Interesting enough, the behavior of the cells cultured on flat substrates and fibril mats of the same material are quite different with respect to the development of focal adhesions, cell cito-skeleton, and cell phenotype [4]. Thus the interest in the technology required for producing nanofiber mats of particular polymeric materials, both of natural or synthetic origin is growing. Nanofiber membranes displaying morphological similarities to the natural extracellular matrix (ECM) characterized by ultrafine continuous fibers, high surface-tovolume ratio, high porosity and variable pore-size distribution similar to the dimensions of basement membranes [5] are then required to exploit the potential of materials in tissue engineering strategies.

Electrospinning is a suitable technique to produce nanofibers due to its easy manufacturing, which is governed by several process parameters that can be controlled by the user $[6,7]$. In the electrospinning process, a solution of the polymeric material in 
an appropriate solvent is projected from a metallic needle to an electrically conductive collector by the action of a high electric field applied by a direct current high voltage power supply (up to $30 \mathrm{kV}$ ). The action of the electric field forms a jet that travels between the needle and the collector evaporating, at least partially, the solvent and precipitating submicron or nano-fibers on the collector surface. Depending on the collector configuration, both oriented and unoriented electrospun mats can be obtained [8-10].

Chitosan finds application in different fields [11-13], in particular in the form of nanofiber mats, due to its chemical structure, similar to glycosaminoglycans (GAGs) which are natural components of the extracellular matrix (ECM) [14]. Chitosan is the deacetylated derivative of chitin, a natural polysaccharide found primarily in the exoskeletons of arthropods and in the cellular wall of some fungi [15].When the degree of chitin deacetylation reaches about $50 \%$ (depending on its origin), it becomes soluble in aqueous media and is called chitosan [16]. Chitosan is composed of residues of glucosamine and $\mathrm{N}$-acetyl glucosamine connected via a-b (1-4) linkage. The ratio of glucosamine and $\mathrm{N}$-acetyl glucosamine is referred as the degree of deacetylation [17]. The solubilization of this polymer occurs by protonation of the $-\mathrm{NH}_{2}$ function on the C2 position of the D-glucosamnie repeat unit, whereby the polysacharide is converted to a polyelectrolyte in acid media. Thus, chitosan is readily soluble in diluted acidic solutions below $\mathrm{pH} 6.0$ [18].

The main problem for the electrospinning of chitosan is its poor solubility. In this way, it is difficult to find a good solvent with the required properties for the electrospinning process [19]. However, several protocols have been experimented with and can found be in the literature [20-22]. Rose et al. [23] reported that trifluoroacetic acid is able to form homogeneous chitosan solutions with good flow properties. Several characteristics 
of this solvent make it suitable for chitosan electrospinning, such as its volatility, dielectric constant and its ability to form salts with the cationic amino groups of chitosan, thereby destroying the rigid interactions between the neighboring chitosan molecules. Gen et al. [23] obtained a 7\% chitosan solution in aqueous $90 \%$ acetic acid solution and could produce electrospun nanofibers. Homayoni et al. [24] also reported the production of electrospun nanofibers with solutions of alkali treated chitosan in aqueous $70-90 \%$ acetic acid. More extensive studies were carried out by Vrieze et al. [25], which reported solutions of chitosan with 75-85\% degree of acetylation in formic acid, acetic acid, lactic acid and hydrochloric acid. In order to improve chitosan solubility other authors reported the addition of co-solvent, such as dicloromethane or methylene chloride $[19,26]$.

Despite the aforementioned effort, more systematic work in order to obtain chitosan nanofibers of the desired size is required. The goal of this paper is to study and evaluate which parameters of the electrospinning process have the strongest influence on fiber thickness in electrospun chitosan mats. For this purpose what is known as Taguchi's method was used [27-29]. The main advantage of this methodology is the possibility to handle with a larger numbers of control parameters with relatively small amount of trials [30-32].

\section{EXPERIMENTAL}

\section{Materials}

All materials were used as received from the provider. Chitosan polymer was purchased from Sigma-Aldrich (viscosity >200.000 cps) with $75 \%$ degree of D-acetylation. Dichloromethane (DCM) and Trifluoroacetic acid (TFA, 99\% ReagentPlus) were purchased from Sigma-Aldrich. 


\section{Preparation of the Polymer Solution}

Solutions of 8, 10 and $12 \%(\mathrm{w} / \mathrm{v})$ chitosan were prepared in TFA:DCM at two different weight ratios $(80: 20$ or $90: 10)$. The solutions were prepared under constant mechanical stirring (Selecta Multimatic 5S) in a heating-bath at $70^{\circ} \mathrm{C}$ until completely dissolution occurred, and then at room temperature for a further 48 hours.

\section{Taguchi's Orthogonal Design}

There are two characteristics of the fibrils that form the electrospun mats which are expected to be important in biological applications. One of them is the fibril diameter: in the present experimental design the aim is to obtain fibers which are as thin as possible. Another relevant characteristic is the dispersion of the fiber diameter sizes, since jet instabilities produce the superposition of thin and thick fibers in the mat. Taguchi's orthogonal design was performed with the aim of determining the experimental parameters most influencing on fiber diameter and diameter dispersion. The effect of five experimental parameters was investigated: polymer concentration in the solution, electric field, needle to collector distance, flow rate and TFA:DCM ratio. Three or two levels were considered for each parameter. Three levels were assigned to polymer concentration $(8,10$ and $12 \% \mathrm{w} / \mathrm{v})$, as well as to the electric field $(20,25$ and $30 \mathrm{kV})$, needle to collector distance $(10,15$ and $20 \mathrm{~cm})$ and flow rate $(1.2,3.6$ and 6.0 $\mathrm{mL} / \mathrm{h}$ ), but only two levels in the case of solvent ratio (80:20 and 90:10). The combination of parameters and levels resulted in the selection of the L18 array proposed for Taguchi (Table 1). 
TABLE 1 Orthogonal table L18 design and mean value $(\overline{\boldsymbol{x}})$ and standard deviation $(\sigma)$ of the diameter of the obtained fibers.

\begin{tabular}{ccccccrr}
$\begin{array}{c}\text { Experiment } \\
\text { Number }\end{array}$ & $\begin{array}{c}\text { Concentration } \\
(\mathbf{w t} \%)\end{array}$ & $\begin{array}{c}\text { Ratio } \\
\text { TFA/DM }\end{array}$ & $\begin{array}{c}\text { Voltage } \\
(\mathbf{k V})\end{array}$ & $\begin{array}{c}\text { Flow } \\
\text { Rate } \\
(\mathbf{m L} / \mathbf{h})\end{array}$ & $\begin{array}{c}\text { Distance } \\
(\mathbf{c m})\end{array}$ & $\begin{array}{c}\overline{\boldsymbol{x}} \\
(\mathbf{n m})\end{array}$ & $\begin{array}{c}\boldsymbol{\sigma} \\
(\mathbf{n m})\end{array}$ \\
\hline $\mathbf{1}$ & 8 & $90 / 10$ & 20 & 1.2 & 10 & 120 & 60 \\
$\mathbf{2}$ & 8 & $90 / 10$ & 25 & 3.6 & 15 & 450 & 210 \\
$\mathbf{3}$ & 8 & $90 / 10$ & 30 & 6.0 & 20 & 480 & 320 \\
$\mathbf{4}$ & 10 & $90 / 10$ & 20 & 1.2 & 15 & 500 & 210 \\
$\mathbf{5}$ & 10 & $90 / 10$ & 25 & 3.6 & 20 & 820 & 510 \\
$\mathbf{6}$ & 10 & $90 / 10$ & 30 & 6.0 & 10 & 79 & 28 \\
$\mathbf{7}$ & 12 & $90 / 10$ & 20 & 3.6 & 10 & 430 & 200 \\
$\mathbf{8}$ & 12 & $90 / 10$ & 25 & 6.0 & 15 & 380 & 130 \\
$\mathbf{9}$ & 12 & $90 / 10$ & 30 & 1.2 & 20 & 930 & 440 \\
$\mathbf{1 0}$ & 8 & $80 / 20$ & 20 & 6.0 & 20 & 550 & 320 \\
$\mathbf{1 1}$ & 8 & $80 / 20$ & 25 & 1.2 & 10 & 216 & 92 \\
$\mathbf{1 2}$ & 8 & $80 / 20$ & 30 & 3.6 & 15 & 270 & 130 \\
$\mathbf{1 3}$ & 10 & $80 / 20$ & 20 & 3.6 & 20 & 540 & 150 \\
$\mathbf{1 4}$ & 10 & $80 / 20$ & 25 & 6.0 & 10 & 310 & 260 \\
$\mathbf{1 5}$ & 10 & $80 / 20$ & 30 & 1.2 & 15 & 220 & 200 \\
$\mathbf{1 6}$ & 12 & $80 / 20$ & 20 & 6.0 & 15 & 430 & 160 \\
$\mathbf{1 7}$ & 12 & $80 / 20$ & 25 & 1.2 & 20 & 1240 & 430 \\
$\mathbf{1 8}$ & 12 & $80 / 20$ & 30 & 3.6 & 10 & 390 & 150 \\
\hline
\end{tabular}

\section{Electrospinning}

The electrospinning apparatus consisted of a high-voltage power supply (Glassman High Voltage Inc, PS/FC 30P04 Model) with a maximal voltage of $30 \mathrm{kV}$. The polymer solution was added in a $10 \mathrm{~mL}$ syringe (medical use) with a needle with $0.5 \mathrm{~mm}$ nozzle diameter. The syringes were placed in a syringe pump (NE-1600 of New Era Pump Systems) and the fibers were collected on a flat aluminum foil placed at a variable distance from the needle (Figure 1). 


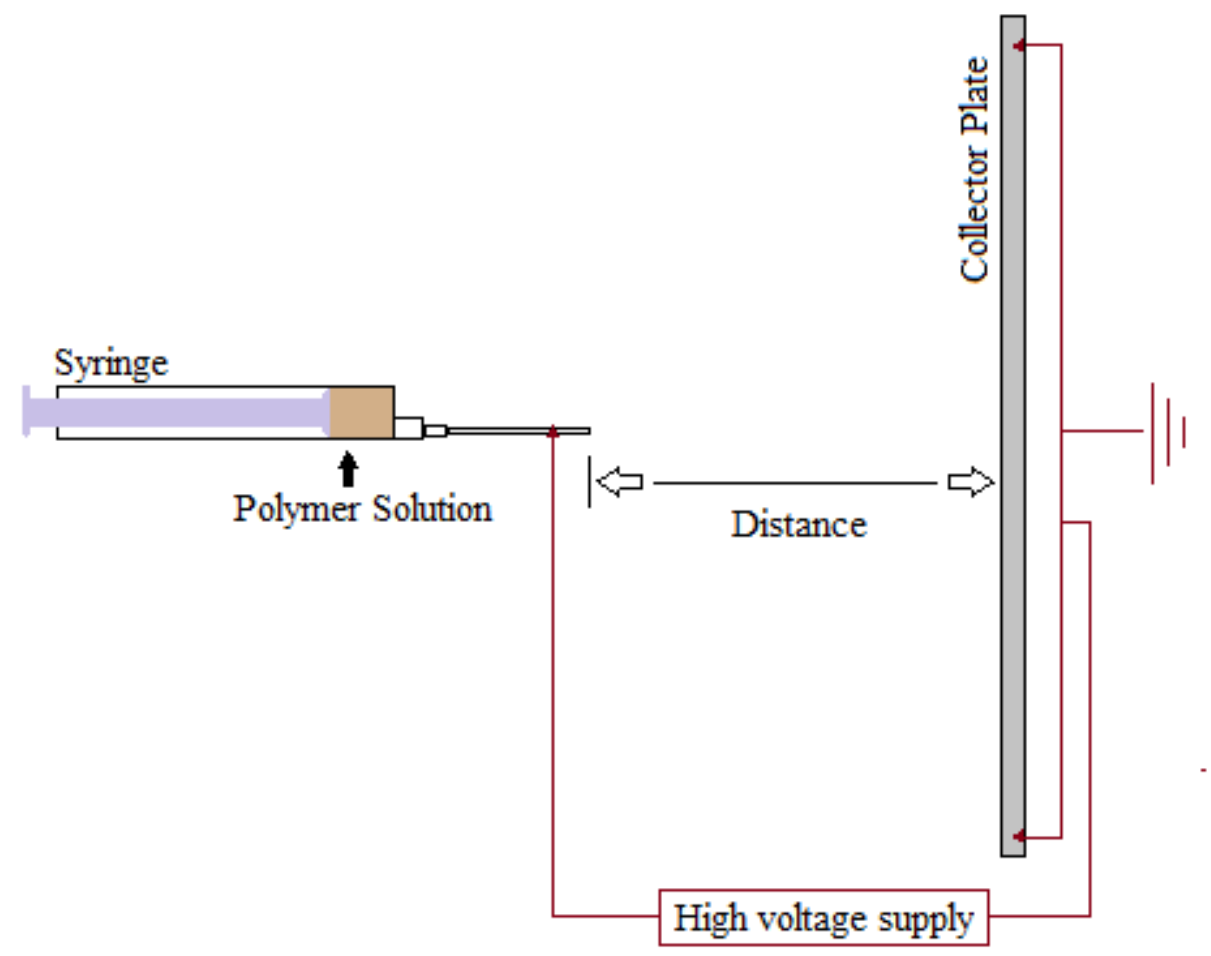

Figure 1. Schematic diagram of electrospinning set-up.

\section{Fiber diameter characterization}

The electrospun fibers were characterized by means of a scanning electron microscope (Model JSM-5410, JEOL with accelerating voltage of $20 \mathrm{kV}$ ) and the image analyzing software ImageJ. The fiber diameters were measured from multiple SEM images, analyzing 100 fibers per experiment. To obtain the diameter of each fiber, an average of 3 measurements on different points of the fiber was taken. The mean diameter and standard deviation were calculated.

\section{Fourier Transform Infrared Spectroscopy}

FTIR spectra for bulk chitosan and chitosan nanofibers were performed at room temperature in a Perkin-Elmer Spectrum 100 apparatus in ATR mode from 4000 to 650 $\mathrm{cm}^{-1}$. FTIR spectra were measured with 32 scans and a resolution of $4 \mathrm{~cm}^{-1}$. 


\section{RESULTS AND DISCUSSION}

The first experiments performed in the present work used trifluoroacetic acid (TFA) as a pure solvent system [33]. TFA is a strong acid that can easily dissolve the polymer through the formation of salts that destroy the hard interactions between the chitosan molecules [20]. Salt formation occurs between the TFA and the amino groups along the chitosan chain and follows the following sequential steps: first, protonation of amine groups $\left(-\mathrm{NH}_{2}\right)$ along the chain of chitosan; second, ionic bonds are formed between protonated amino groups $\left(-\mathrm{NH}_{3}\right)$ and the trifluoroacetate $\mathrm{C}_{2} \mathrm{~F}_{3} \mathrm{O}_{2}$ anions formation (Figure 2) [19, 22]. In this configuration, the salts are soluble in an aqueous mean.

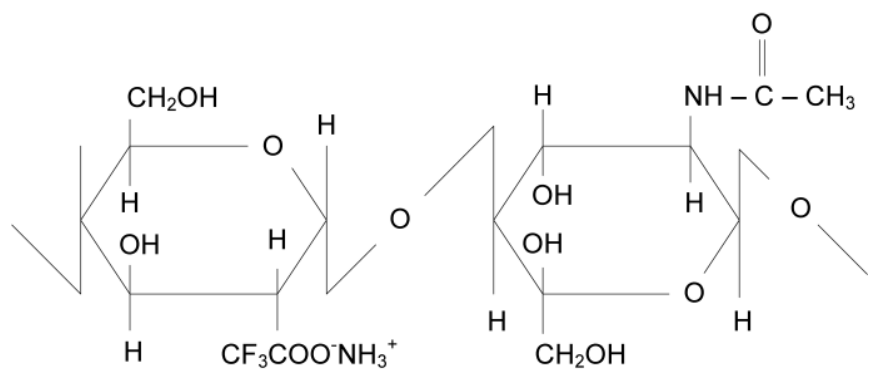

Figure 2. Molecular structure of the chitosan molecule after the dissolution in TFA.

However, fiber production proved to be hard and non-continuous. The presence of beads was constant, which destroyed the fiber mats. Sometimes, the electrospinning process apparently took placed without any problem but the thin layer deposited in the aluminum sheet did not display the expected nanofibers: instead, beads were deposited. In this case, the process taking place was electrospraying and can be explained by the repulsive interactions between the polycations along the chitosan chains, which consequently did not reach sufficient chain entanglements for fiber formation.

As a solution, a new solvent was added. The Dicloromethane (DCM) in a TFA solution has the ability to decrease the dielectric constant once the dielectric constant of TFA is 
quite high $\left(\varepsilon_{\mathrm{TFA}} \sim 30\right.$ at $20^{\circ} \mathrm{C}$ and $\varepsilon_{\mathrm{DCM}} \sim 9.1$ at $\left.20^{\circ} \mathrm{C}\right)$ [34]. $\mathrm{DCM}$ also reduces the density in the solution originated by the TFA and also generates better electrospinnability conditions. Nevertheless, some beads still appear in the electrospun nanofibers. Three different ratios $-90: 10,80: 20$ and 70:30 - were selected. The 70:30 ratio was not used due to the difficulty of dissolving the polymer. Furthermore, the solution ratio 70:30 also displayed bad electrospinnability (clogging the needle and the jet dropping). For the 90:10 and 80:20 ratio, electrospun mats could not be obtained with a solution concentration of less than $8 \mathrm{wt} \%$.

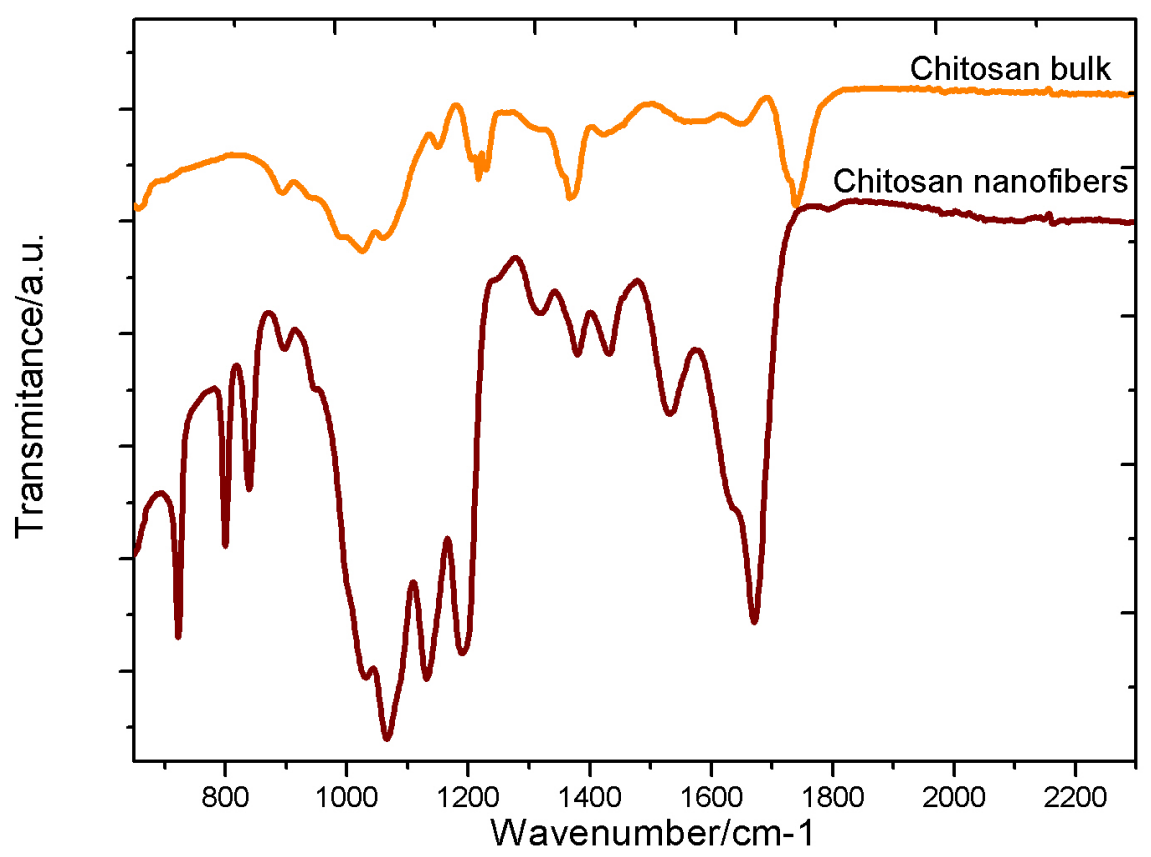

Figure 3. FT-IR spectra of bulk chitosan and electrospun chitosan nanofibrous membranes. 
Figure 3 shows a comparison between the FT-IR spectra of the electrospun chitosan nanofiber membranes and those of bulk chitosan (as received, in powder form). The characteristic absorption peaks of bulk chitosan can be observed by the combination of amide I bands at 1655 and $1630 \mathrm{~cm}^{-1}$, amide II band at $1543 \mathrm{~cm}^{-1}$, the bridge oxygen stretching band at $1160 \mathrm{~cm}^{-1}$, and the C-O stretching bands at 1070 and $1030 \mathrm{~cm}^{-1}$ [35]. In addition, due to the fact that chitosan has a $75 \%$ degree of $\mathrm{D}$-acetylation, the peak of the amino group at $1595 \mathrm{~cm}^{-1}$ can also be also observed [36]. The presence of a large absorption peak at $1675 \mathrm{~cm}^{-1}$ and the three absorption peaks around $840-720 \mathrm{~cm}^{-1} \mathrm{can}$ be observed as a consequence of the presence of trifluoroactic acid in the nanofibers and the amino salts as explained above. At 1675 and $1530 \mathrm{~cm}^{-1}$ it is possible to observe the peaks corresponding to the stretching of the protonated amino $\left(-\mathrm{NH} 3^{+}\right)$groups (Figure 3) $[19]$.

It is worth to notice that the obtained electrospun mat is soluble in water. To use the electrospun chitosan nanofiber membranes in applications that require a contact with an aqueous medium, previous regeneration of the amino groups in the electrospun nanofibers is required. This process was successfully performed by immersing the mats in a saturated $\mathrm{Na}_{2} \mathrm{CO}_{3}$ aqueous solution with an excess amount of $\mathrm{Na}_{2} \mathrm{CO}_{3}$ (s) in the solution [19]. 


\section{Fiber Morphology}
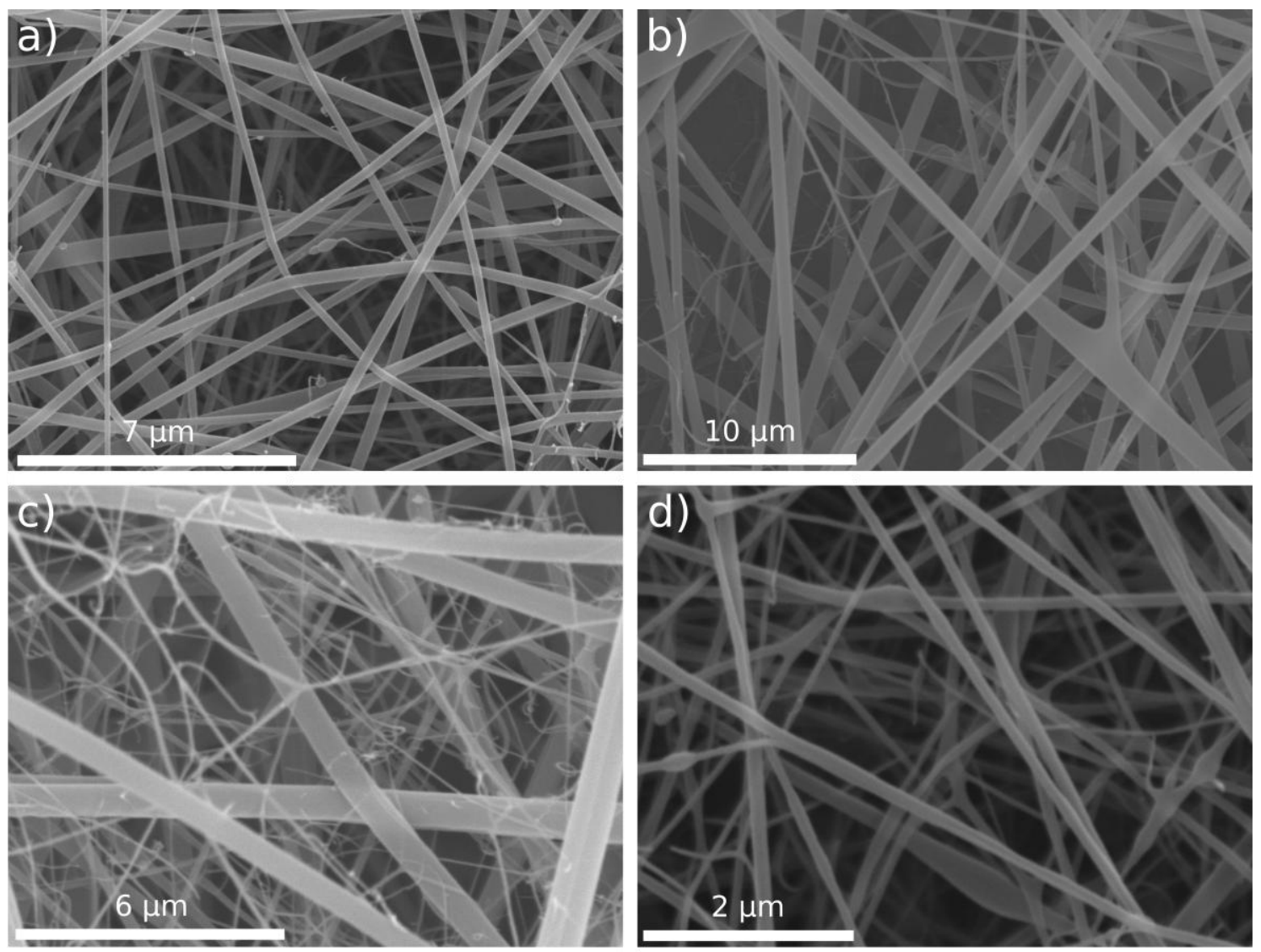

Figure 4. SEM photographs of several electrospun membranes obtained with the experimental conditions selected in Taguchi's design and listed in Table 2. The pictures correspond to experiments 11 (a); 4 (b); 14 (c); and 6 (d) of Table 1.

Processing parameters have a large influence on the shape, distribution and size of the produced chitosan fibrils. Figure 4 shows a selection of SEM photographs of the electrospun mats obtained in the 18 experiments (Table 1). The selected pictures represent the different morphologies obtained. Figure 4a (experiment 11) shows a mat consisting of randomly oriented, highly homogeneous nanofibers (the mean diameter in this sample is $215 \mathrm{~nm}$ ). Nevertheless, nanofiber defects can be observed, such as 
branched fibers (Figure 4b), substructures of smaller fibers (Figure 4c), and beads on the fibers (Figure $4 d$ ).

However, with different sets of processing parameters, mats with broad distributions of fiber diameters are obtained, as shown in Figure 4 [8]. In Figures $4 \mathrm{~b}$ and $4 \mathrm{~d}$ two different fiber microstructures are obtained: thin and thick nanofibers. Furthermore, the small net of nanofibers is not continuous. This feature is to be attributed to viscosity. Due to the fast evaporation of the solvent, the solution has a high amount of polymer and low acid concentration, and therefore a higher viscosity. In this way, it is necessary to clean the tip of the needle several times during the process, inducing jet initialization, in order to obtain the observed microstructure. It should be remarked that in this situation the jet instability increases due to the variation in viscosity, an effect which is even reinforced due to the high voltage applied $(25 \mathrm{kV})$.

In other cases the distribution of fiber diameter becomes clearly bimodal around the two most frequent values, the mat therefore consisting of intermingled thick and thin fibers, as shown in Figures $4 \mathrm{~b}$ and $4 \mathrm{c}$. The presence of sub-structures of smaller fibers between smooth large fibers suggests the formation of a secondary jet during the main electrospinning procedure due to high solution viscosity. Ding et al. [37] pointed out that their occurrence was related to certain process conditions such as high voltage, low relative humidity and fast phase separation of polymer and solvent during the flight between the needle and the collector.

In Figure 4b branched fibers can be observed. Ramakrishna et al. [8] justified these structures as a consequence of the formation of smaller jets ejected from the surface of the primary jets, comparable to the ejection of an initial jet from the surface of a charged droplet. It is proposed that the elongation of the jet and evaporation of the solvent modify the shape and the charge density of the jet. Thus, the balance between 
the electrical forces and surface tension can shift, thereby causing the shape of a jet to be unstable. Such instability can decrease its local charge per unit surface area by ejecting a smaller jet from the surface of the primary jet or by splitting apart into two smaller jets.

The presence of small globules on the surface of the electrospun fibers in some experiments has been explained by many authors as a consequence of the presence of salts [38]. As explained above, the presence of salts between TFA and amino groups can be the reason for the presence of these small structures. The sample showing these kind of structures has a concentration of $12 \mathrm{wt} \%$ in a ratio of 90:10 (v/v \%), which corresponds to the maximum concentration used in this experimental set. With the formation of salts, the conductivity of the solution also increases and consequently the net charge density carried by the jet in electrospinning process is affected.

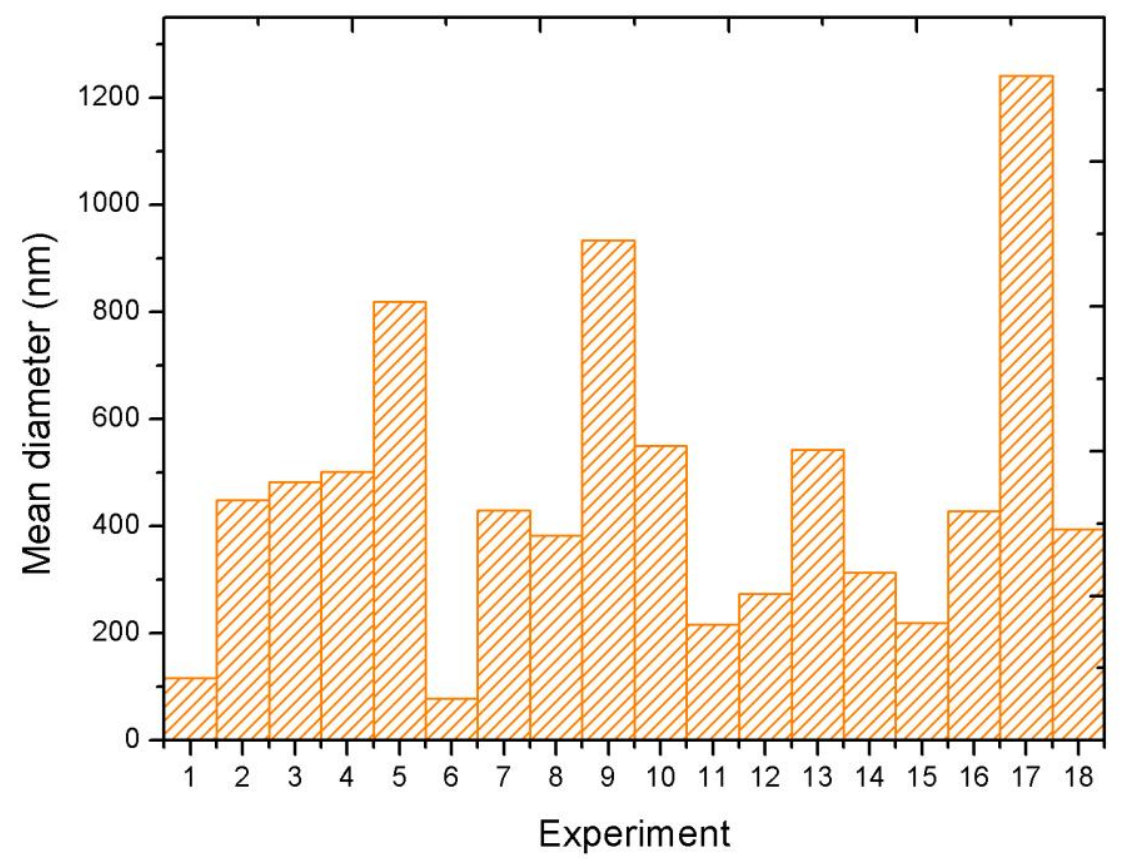

Figure 5. Mean diameter distribution of chitosan nanofibers in the Taguchi experiments. 
However, experiment number 9, which has the same solution properties, did not display the same defects, indicating that other parameters may have an influence in the formation of the globules too. Figure 5 shows the average fiber diameter in the 18 experiments. This quantitatively supports the discussion in the former paragraphs. The mean diameter varies between $79 \mathrm{~nm}$ in experiment 6 and 1240 in experiment 17 . The plot in Figure 5 shows how difficult it is to determine the influence of any individual process parameter on fiber morphology, since all parameters are highly correlated.

TABLE 2 Influence of different levels of each parameter on the fiber diameter (FD) and its standard deviation (SD)

\begin{tabular}{|c|c|c|c|c|c|c|}
\hline $\begin{array}{c}\text { FD } \\
(\mathbf{n m})\end{array}$ & & Concentration & Voltage & Distance & Flow Rate & $\begin{array}{c}\text { Ratio } \\
\text { TFA/DCM }\end{array}$ \\
\hline & Level 1 & 348 & 428 & 258 & 538 & 466 \\
\hline & Level 2 & 412 & 570 & 375 & 484 & 464 \\
\hline & Level 3 & 632 & 397 & 761 & 372 & \\
\hline & Range & 287 & 173 & 503 & 165 & 2 \\
\hline & Importance & 2 & 3 & 1 & 4 & 5 \\
\hline & Best level & $8 \mathrm{wt} \%$ & $30 \mathrm{kV}$ & $10 \mathrm{~cm}$ & $6.0 \mathrm{~mL} / \mathrm{h}$ & $80-20$ (v/v \%) \\
\hline \multirow{7}{*}{$\begin{array}{c}\text { SD } \\
(\mathbf{n m})\end{array}$} & & Concentration & Voltage & Distance & Flow Rate & $\begin{array}{c}\text { Ratio } \\
\text { TFA/DCM }\end{array}$ \\
\hline & Level 1 & 189 & 185 & 134 & 265 & 234 \\
\hline & Level 2 & 227 & 272 & 173 & 228 & 212 \\
\hline & Level 3 & 253 & 211 & 362 & 203 & \\
\hline & Range & 63 & 87 & 228 & 61 & 21 \\
\hline & Importance & 3 & 2 & 1 & 4 & 5 \\
\hline & Best level & $8 \mathrm{wt} \%$ & $20 \mathrm{kV}$ & $10 \mathrm{~cm}$ & $3.6 \mathrm{~mL} / \mathrm{h}$ & $80-20(\mathrm{v} / \mathrm{v} \%)$ \\
\hline
\end{tabular}

Taguchi's method makes it possible to determine which parameters have the greatest influence on the mean fiber diameter and on the standard deviation of the fiber diameter, within the range of variation between the different levels assigned in the experimental design. Thus, as shown in Table 2, the distance from the needle to the collector is the most influent parameter both on mean fiber diameter and on fiber homogeneity. A longer distance from the collector (from 10 to $20 \mathrm{~cm}$ in our experiments) yields thicker and less homogeneous fibers. Furthermore, the changes in 
fiber diameter and standard deviation due to changes in the travelling distance are more important than those produced by changes in the rest of parameters. The interpretation of this result is not straightforward since changes in the distance between the needle and the collector affect various features of fiber formation. A shorter distance means a higher value of the electric field that produces the jet and can also affect the probability of jet instabilities. On the other hand, travelling distance affects solvent evaporation before the fiber is deposited on the collector. The results obtained lead to the conclusion that the optimal value for this parameter is $10 \mathrm{~cm}$, so as to obtain smaller fiber diameters.

The second influent parameter on mean diameter is the solution concentration. It seems clear than more diluted solutions yield thinner fibers. The evaporation of solvent from the jet solution produces smaller fibers as lower is the polymer content, as reported previously $[30,39,40]$. Chitosan is a cationic polyelectrolyte, and then ion concentration increases with the chitosan content increasing conductivity and also the charge density on the surface of the drop formed in the needle, which increases elongational forces on the jet, hence thinner fiber are expected $[9,41,42]$. On the other hand, as the solution concentration increases also does the viscosity, that produce thicker fibers $[43,44]$. In our case, the combination of both effects results in an increase of fiber diameter as concentration increases, as can be seen in figure 6 . 


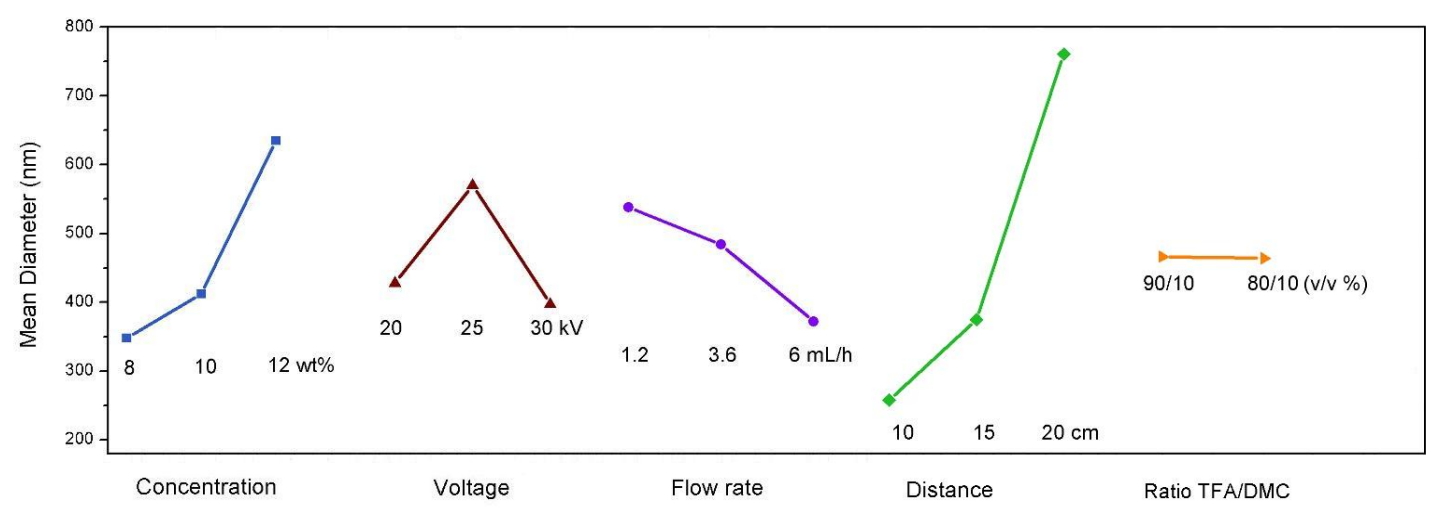

Figure 6. Influence of the different parameters on the fiber diameter.

The influence of concentration on fiber homogeneity is not so important (is the third influent parameter with respect to standard deviation, as shown in Table 2). This means that differences in fiber homogeneity found among the different experiments performed with the same solution concentration, due to changes in the rest of parameters, might be more important than those produced by changes in the concentration itself. We will consider the optimal value of concentration to be $8 \%$ from the results obtained regarding mean fiber diameter.

The effect of the other processing parameters on mean fiber diameter is smaller than those of travelling distance and solution concentration. Their optimal values were selected with the criteria of the minimum average fiber diameter. In the case of the TFA/DCM ratio, the lack of a significant influence must be explained by the narrow range of solvent compositions included in the present experimental design. Solvent ratio is determinant in fiber formation as probed by the fact that out of the interval of DCM content from 10 to $20 \%$ either no fibers are formed or the fibers present a large number of beads or defects, as explained above. 


\section{Optimal Parameter Values}

The conclusion of the analysis is that in order to produce an electrospun mat with the thinnest fiber diameter and the minimum standard deviation, the optimum levels of the process parameters are: concentration $8 \mathrm{wt} \%$, voltage $30 \mathrm{kV}$, distance $10 \mathrm{~cm}$, flow rate $6.0 \mathrm{~mL} / \mathrm{h}$, ratio of TFA:DCM 80:20. These conditions do not correspond to any of the 18 experiments performed, and thus, in order to test the result of the experimental design, electrospun mats were produced with the optimal set of parameters. The result is shown in the SEM pictures in Figure 7. Homogeneous fibers with a mean diameter of $66 \mathrm{~nm}$ were obtained.

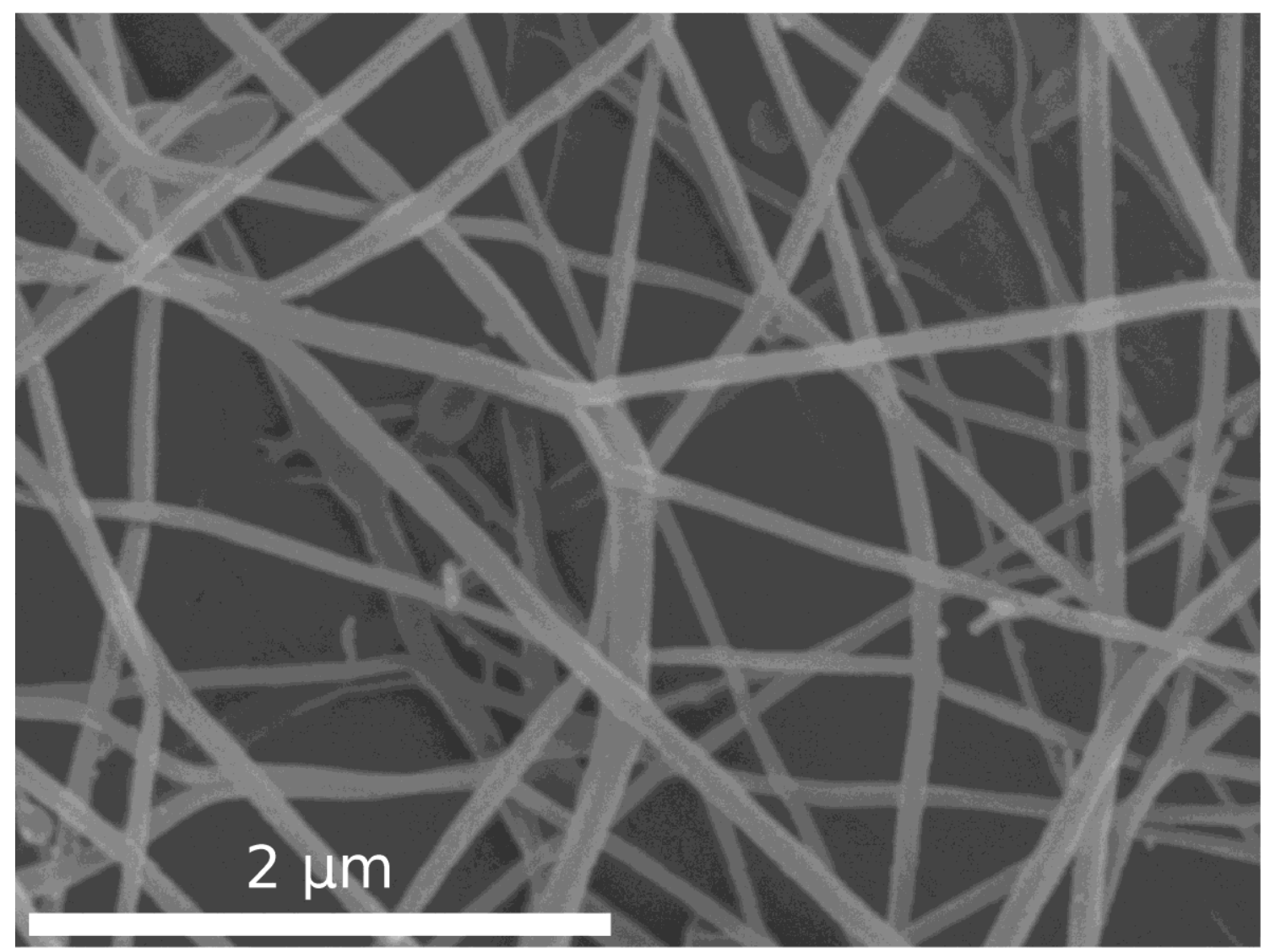

Figure 7. SEM photographs of $8 \mathrm{wt} \%$ of chitosan nanofiber mats electrospun from TFA/DCM 80:20 (v/v \%) with $30 \mathrm{kV}$ of voltage, $6.0 \mathrm{~mL} / \mathrm{h}$ of flow rate, $10 \mathrm{~cm}$ of distance and with a size needle of $0.5 \mathrm{~mm}$. The mean diameter is $66 \mathrm{~nm}$. 
In this way, it was demonstrated that the Taguchi method makes it possible to assess the best parameters to produce optimized nanofiber chitosan mats with tailored fiber diameters and with small diameter standard distribution for specific applications.

\section{CONCLUSION}

Electrospun chitosan nanofiber mats have been obtained using chitosan solutions in a mixture of trifluoroacetic acid and dichloromethane. No processing conditions were found to obtain homogenous fibers from chitosan solutions in pure trifluoroacetic acid. Dichloromethane decreases the dielectric constant of the solution, thus improving electrospinnability.

Uniform fibers were formed only with a narrow window of solvent ratio: we have selected 90:10 and 80:20 TFA:DCM ratios. For higher amounts of dichloromethane, it was very difficult to dissolve the polymer, and the solution displayed bad electrospinnability due to needle clogging and jet dropping.

An experimental design has been performed in order to obtain nanofibers which are as thin and homogeneous as possible. Taguchi's approach for selection of process parameters made it possible to produce nanofibers with an average diameter of $66 \mathrm{~nm}$ in the form of a randomly oriented mat.

The most influent parameter on fiber diameter and fiber homogeneity was the distance from the needle to the collector. A shorter distance to the collector yields thinner and more homogeneous fibers.

The second influent parameter on mean diameter is solution concentration. The evaporation of solvent in jet travel produces smaller fibers as the polymer content in the solution decreases. Furthermore, as the solution concentration increases, so does 
viscosity, which results in thicker fibers. The combination of both effects results in a decrease in fiber diameter as concentration decreases.

The influence of applied voltage, feeding rate and solvent composition are less significant that the rest of parameters.

The best parameters obtained after Taguchi optimization for thinner fiber formation are a concentration of $8 \mathrm{wt} \%$, a TFA/DCM ratio of 80:20 (v/v \%), a voltage of $30 \mathrm{kV}$, a flow rate of $6.0 \mathrm{~mL} / \mathrm{h}$ and a gap distance of $10 \mathrm{~cm}$.

\section{ACKNOWLEDGEMENTS}

SLM and VS, would like to thank the Portuguese Foundation for Science and Technology (FCT) Grant NANO/NMed-SD/0156/2007 and VS the FCT for the SFRH/BPD/63148/2009 grant. JLGR acknowledges the support of the Spanish Ministry of Science and Innovation through project No. MAT2010-21611-C03-01 (including the FEDER financial support) and Programa Nacional de Internacionalización de la I+D project EUI2008-00126. Funding for research in the field of Regenerative Medicine through the collaboration agreement from the Conselleria de Sanidad (Generalitat Valenciana), and the Instituto de Salud Carlos III (Ministry of Science and Innovation) is also acknowledged. This study has been also supported by a grant from the Spanish Ministry of Science and Innovation, Centro para el Desarrollo Tecnológico Industrial (CDTI), CENIT: “Customized Eye Care”, CeyeC (CEN-20091021). The translation of this paper was funded by the Universitat Politècnica de València, Spain. 


\section{REFERENCES}

1. M. Veerapandian and K. Yun, Dig. J. Nanomater. Bios., 4, 243 (2009).

2. B.B. Ward, S.E. Brown, and P.H. Krebsbach, Oral Dis., 16, 709 (2010).

3. F. Destro, M. Borgatti, B. Iafelice, R. Gavioli, T. Braun, J. Bauer, L. Böttcher, E. Jung, M. Bocchi, R. Guerrieri, and R. Gambari, J. Mater Sci.-Mater. M., 21, 2653 (2010).

4. M. Deng, L.S. Nair, S.P. Nukavarapu, S.G. Kumbar, T. Jiang, A.L. Weikel, N.R. Krogman, H.R. Allcock, and C.T. Laurencin, Adv. Funct. Mater., 20, 2794 (2010).

5. D. Patel, M.M. Patel, M.P. Patel, and N.M. Patel, J. Pharm. Res., 2, 1234 (2009).

6. A. Formhals, U.S. Patent, 1975504 (1934).

7. A. Formhals, U.S. Patent, 6641773 (1940).

8. S. Ramakrishna, K. Fujihara, W. E. Teo, T. C. Lim, and Z. Ma, Introduction to Electrospinning and Nanofibers. Sinngapore: World Scientific, (2005).

9. X. Zong, K. Kim, D. Fang, S. Ran, B.S. Hsiao, and B. Chu, Polymer, 43, 4403 (2002).

10. U. Boudriot, R. Dersch, A. Greiner, and J.H. Wendorff, Artif. Organs, 30, 785 (2006).

11. I. Arvanitoyannis, I. Kolokuris, A. Nakayama, N. Yamamoto, and S.-i. Aiba, Carbohydr. Polym., 34, 9 (1997).

12. I.S. Arvanitoyannis, A. Nakayama, and S.-i. Aiba, Carbohydr. Polym., 37, 371 (1998).

13. I.S. Arvanitoyannis, J. Macromol. Sci. - Rev. Macromol. Chem. Phys., 39, 205 (1999). 
A. C. Areias et al., Polymer Engineering And Science, 52 (6): 1293-1300, 2013

14. Hsi-Yi Yeh and J.-C. Lin, J. Biomat Sci-Polym. E., 19, 291 (2008).

15. M. Rinaudo, Prog. Polym. Sci., 31, 603 (2006).

16. C.K.S. Pillai, W. Paul, and C.P. Sharma, Prog. Polym. Sci., 34, 641 (2009).

17. M.R. Kasaai, J. Agr. Food Chem., 57, 1667 (2009).

18. K.V. Harish Prashanth and R.N. Tharanathan, Trends Food Sci. Tech., 18, 117 (2007).

19. P. Sangsanoh and P. Supaphol, Biomacromolecules, 7, 2710 (2006).

20. K. Ohkawa, D. Cha, H. Kim, A. Nishida, and H. Yamamoto, Macromol. Rapid Comm., 25, 1600 (2004).

21. A. Neamnark, R. Rujiravanit, and P. Supaphol, Carbohyd. Polym., 66, 298 (2006).

22. J.D. Schiffman, L.A. Stulga, and C.L. Schauer, Polym. Eng. Sci., 49, 1918 (2009).

23. X. Geng, O.-H. Kwon, and J. Jang, Biomaterials, 26, 5427 (2005).

24. H. Homayoni, S.A.H. Ravandi, and M. Valizadeh, Carbohyd. Polym., 77, 656 (2009).

25. S. De Vrieze, P. Westbroek, T. Van Camp, and L. Van Langenhove, J. Mater. Sci., 42, 8029 (2007).

26. W. Wang, S. Itoh, K. Konno, T. Kikkawa, S. Ichinose, K. Sakai, T. Ohkuma, and K. Watabe, J. Biomed. Mater. Res. A, 91A, 994 (2009).

27. G. Taguchi, The System of Experimental Design: Engineering Methods to Optimize Quality and Minimize Costs. New York: Unipub/Kraus International Publications, (1987).

28. G. Taguchi and S. Konishi, Taguchi Methods: Orthogonal Arrays and Linear Graphs. New York: American Supplier Institute, (1987). 
A. C. Areias et al., Polymer Engineering And Science, 52 (6): 1293-1300, 2013

29. S. Maghsoodloo, G. Ozdemir, V. Jordan, and C.-H. Huang, J. Manuf. Syst., 23, 73 (2004).

30. P. Heikkilä and A. Harlin, Eur. Polym. J., 44, 3067 (2008).

31. J.A. Gómez-Tejedor, N.V. Overberghe, P. Rico, and J.L.G. Ribelles, Eur. Polym. J., 47, 119 (2011).

32. P.N. Honkhambe, C.V. Avadhani, P.P. Wadgaonkar, and M.M. Salunkhe, J. Appl. Polym. Sci., 106, 3105 (2007).

33. J.D. Schiffman and C.L. Schauer, Biomacromolecules, 8, 594 (2006).

34. B. Veleirinho, M.F. Rei, and J.A. Lopes-Da-Silva, J. Polym. Sci. Pol. Phys., 46, 460 (2008).

35. J.D. Schiffman and C.L. Schauer, Biomacromolecules, 8, 2665 (2007).

36. I. Yamaguchi, S. Itoh, M. Suzuki, A. Osaka, and J. Tanaka, Biomaterials, 24, (2003).

37. B. Ding and et al., Nanotechnology, 17, 3685 (2006).

38. C. Zhang, X. Yuan, L. Wu, Y. Han, and J. Sheng, Eur. Polym. J., 41, 423 (2005).

39. A. Koski, K. Yim, and S. Shivkumar, Mater. Lett., 58, 493 (2004).

40. A.K. Haghi and M. Akbari, phys. stat. sol. (a), 204, 1830 (2007).

41. K.-H. Zhang, Q.-Z. Yu, and X.-M. Mo, Int. J. Mol. Sci, 12, 2187 (2011).

42. P. Chuysinuan, N. Chimnoi, S. Techasakul, and P. Supaphol, Macromol. Chem. Physic. , 210, 814 (2009).

43. P.K. Baumgarten, J. Colloid Interface Sci., 36, 71 (1971).

44. J.-H. He, Y.-Q. Wan, and J.-Y. Yu, Fibers Polym., 9, 140 (2008). 